\title{
Design and Implementation of Fractional Order Controller for Service Robots
}

\author{
Tang Qingshun ${ }^{1}$, Wu Chunfu ${ }^{1}$, Yang Yuanhui ${ }^{1}$, Li Guodong ${ }^{1}$ and Zhou Fengyu ${ }^{2}$ \\ ${ }^{1}$ School of Physics, Mechanical and Electrical Engineering, Longyan University, \\ Longyan China \\ ${ }^{2}$ School of Control Science and Engineering, Shandong Univer-sity, Jinan, China \\ Qingshun951128@163.com
}

\begin{abstract}
In this paper, the fractional order motion control is designed basing on the Leader I$D X$ service robotic platform, where the implementation of robust fractional order controller is introduced in details. The fractional order PD $\mu$ control is analyzed in frequency domain by using an FIR/IIR method. The system model of the motor of Leader $I-D X$ is established in MATLAB so that the influence of $\mu$ to the whole control system can be verified, and the comparisons of $P D$ and $P D \mu$ controllers are shown as well. Motivated by the better performance of fractional order controllers, a digital implementation of fractional order $P D \mu$ controllers using PRONY technique is applied so that the incremental fractional order controllers can be realized. The real experiments on Leader I-DX platform are illustrated to validate the above concepts.
\end{abstract}

Keywords: Service robot; Motion control; Fractional calculus; Fractional order PD $\mu$ controller

\section{Introduction}

With the development of society and the progress of science and technology, the research and applications of service robots have been paid more attention than ever before, and it is gradually emerged into people's lives. It is well known that the motion performance is an important index to measure the robots. The high precision control of robots are strongly relied on the reliability of control systems, and the advanced motion control strategy. At present, in the process of designing the robot controller, the PID algorithm is the most widely used one that has simple structure, strong adaptability, good robustness and anti-perturbation ability, etc. However, the traditional PID algorithm may not be ideal in the control of nonlinear, time-varying, coupling and other complex systems. Thus the PID algorithm cannot meet the requirements of high performance robots in many respects.

The history of fractional calculus is the same long as the traditional calculus, which adopts the derivatives and integrals with arbitrary real or even complex numbers [1-2]. It has been pointed in [3] that "the fractional order system is ubiquitous in reality". Besides, it can be seen that the fractional order theory can better reveal the facts of the real systems, therefore it is a better way to system modeling [4-8]. Nowadays, the fractional order control has become an important part of modern control theory and its applications [9-11]. Particularly, the optimal control strategy of fractional order system is always pointing to the fractional order one. It is a meaningful work about the fractional order systems and controls. The CRONE control is proposed by Oustaloup [12]; the fractional order PD control is discussed by Dorcak in [13]; the fractional order $\mathrm{PI}^{\lambda} \mathrm{D}^{\mu}$ control is developed by Podlubny in [14]. Meanwhile, the applications of fractional calculus to various areas are shown in [15-18]. 
In this paper, the fractional order $\mathrm{PD}^{\mu}$ control is applied successfully to the Leader I-DX robot [19]. It will be shown that the fractional order dynamics and controls compensates the properties of the system that are ignored in integer order discussions. Some mathematical preliminaries are introduced in Section 1. The model of Leader I-DX service robot's motor is established in Section 2. In Section 3 a robust fractional order $\mathrm{PD}^{\mu}$ controllers is designed for the motion control of the Leader I-DX service robotic platform. With the established MATLAB simulation model, Section 4 presents the influence of the fractional derivative operator $\mu$ to the control system and the comparisons between $\mathrm{PD}$ and $\mathrm{PD}^{\mu}$ controllers. Section 5 realizes the discretization of the fractional operator and the implementation of fractional order controller. The experimental application results are shown in Section 6. Finally, the conclusions are shown in Section 7.

\section{Fractional Calculus and Fractional Order $\mathbf{P I}^{\lambda} D^{\mu}$ Control}

The Grünwald-Letnicov fractional order derivative is defined as

$$
{ }_{t_{0}} D_{t}^{\alpha} f(t)=\lim _{h \rightarrow 0} h^{-\alpha} \sum_{j=0}^{\left[\frac{t-t_{0}}{h}\right]}(-1)^{j}\left(\begin{array}{c}
\alpha \\
j
\end{array}\right) f(t-j h)
$$

where $h$ is the sample instant, and $[X]$ denotes the integer part of $X$. The continuous Riemann-Liouville (RL) fractional order operator is ${ }_{t_{0}} D_{t}^{\alpha}$, which is shown as

$$
{ }_{\alpha} D_{t}^{\alpha}=\left\{\begin{array}{cc}
\frac{d^{\alpha}}{d t^{\alpha}}, & R(\alpha)>0 \\
1, & R(\alpha)=0 \\
\int_{\alpha}^{t}(d \tau)^{(-\alpha)}, & R(\alpha)<0
\end{array}\right.
$$

Moreover, the integer order PID control strategy is

$$
u(t)=K_{p}\left[e(t)+K_{i} \int_{0}^{t} e(\tau) d \tau+K_{d} \frac{d e(t)}{d t}\right]
$$

that can be extended to the fractional order $\mathrm{PI}^{\lambda} \mathrm{D}^{\mu}$ control:

$$
u(t)=K_{p}\left[e(t)+K_{i} D_{t}^{-\lambda} e(t)+K_{d} D_{t}^{\mu} e(t)\right]
$$

The Laplace transform of RL derivative with zero initial condition is

$$
L\left[{ }_{0} D_{t}^{\alpha} f(t)\right]=s^{\alpha} F(s)
$$

so that the transfer function of fractional order $\mathrm{PI}^{\lambda} \mathrm{D}^{\mu}$ control is shown as

$$
G(s)=\frac{U(s)}{E(s)}=K_{p}\left(1+\frac{K_{i}}{s^{\lambda}}+K_{d} s^{\mu}\right)
$$




\section{The Modeling of Leader I-DX's DC Motor}

The scheme of DC motor is shown in Figure 1, and its transfer function can be denoted as:

$$
G(s)=\frac{\theta(s)}{u(s)}=\frac{1}{s\left(T_{m} s+1\right) k_{e}}=\frac{K}{s\left(T_{m} s+1\right)}
$$

where $K$ is the speed constant, $\mathrm{T}_{m}=\frac{R_{a} J}{k_{e} k_{m}}$ is the time constant of the DC motor.

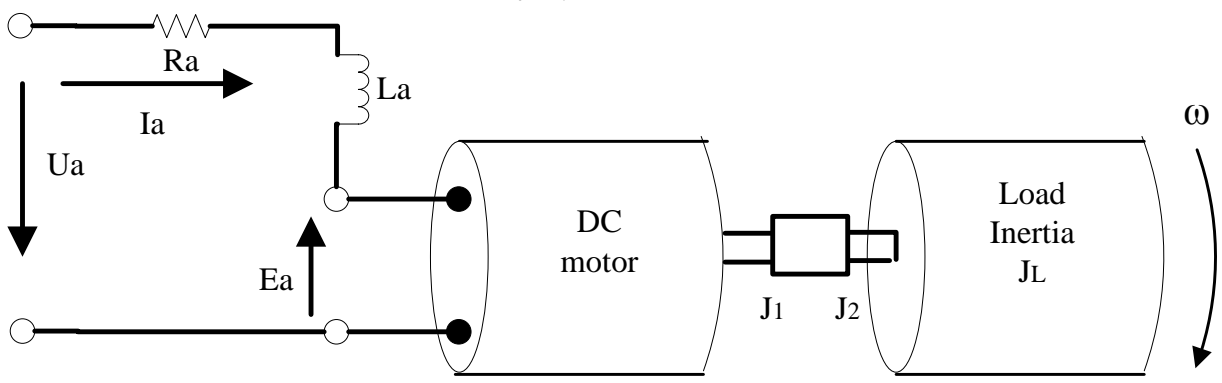

Figure 1. The Scheme of DC Motor

The driven motor of Leader I-DX is the MAXON RE30 DC motor, where $R_{a}=0.198 \Omega$ ; $L_{a}=3.45 \times 10^{-5} \mathrm{H} ; K=685 \mathrm{rpm} / \mathrm{v}=71.73 \mathrm{rad} / \mathrm{v} . \mathrm{s} ; k_{m}=1.39 \times 10^{-2} \mathrm{Nm} / \mathrm{A} ; J_{l}=3.35 \times 10^{-6} \mathrm{Kg} . \mathrm{m}^{2}$ ; $J_{2}=7 \times 10^{-6} \mathrm{Kg} \cdot \mathrm{m}^{2} ; \eta=86 \%$; $\mathrm{i}=66: 1$.

The total mass of Leader I-DX is $m \approx 50 \mathrm{~kg}$, the driving wheel diameter is $D=200 \mathrm{~mm}$, and the load moment of inertia is $\mathrm{J}_{\mathrm{L}}=\frac{m}{2} \cdot \frac{D^{2}}{4}=0.25 \mathrm{Kg} \cdot \mathrm{m}^{2}$. Besides, the motor shaft inertia in total: $J=J_{1}+J_{2}+J_{L} / i^{2} \eta=7 \times 10^{-5} \mathrm{Kg} \cdot \mathrm{m}^{2}$. Based on the above parameters, the time constant of the $\mathrm{DC}$ motor is $\mathrm{T}_{\mathrm{m}}=0.07$.

Therefore, the transfer function of the DC motor is

$$
G(s)=\frac{\theta(s)}{u(s)}=\frac{71.73}{s(0.07 s+1)}
$$

\section{Fractional Order $\mathbf{P D}^{\mu}$ Control}

\subsection{Design Principles of Robust Fractional Order PD ${ }^{\mu}$ Controller}

In the design of robust controller, analyze the control system based on the desired amplitude margin and phase margin from the aspect of relative stability in frequency domain can meet the requirements of the system. In this paper, the fractional order controller is designed based on the amplitude margin and phase margin.

It follows from (6) that the fractional order $\mathrm{PD}^{\mu}$ controller can be expressed as

$$
C(s)=K_{P}\left(1+K_{d} s^{\mu}\right)
$$

where $\mu \in(0,1]$. Obviously, (9) is a special case of fractional order $\mathrm{PI}^{\lambda} \mathrm{D}^{\mu}$ control where $\lambda=0$.

The magnitude and phase characteristics of DC motor are 


$$
\begin{gathered}
\arg |G(j \omega)|=-\arctan \left(\omega T_{m}\right)-\frac{\pi}{2} \\
|G(j \omega)|=\frac{K}{\omega \sqrt{1+\left(\omega T_{m}\right)^{2}}}
\end{gathered}
$$

Moreover, the $\mathrm{PD}^{\mu}$ controller can be expressed in frequency domain that

$$
C(j \omega)=K_{p}\left[1+K_{d}(j \omega)^{\mu}\right]=K_{p}\left(1+K_{d} \omega^{\mu} \cos \frac{\mu \pi}{2}+j K_{d} \omega^{\mu} \sin \frac{\mu \pi}{2}\right)
$$

where

$$
\begin{gathered}
\arg |C(j \omega)|=\arctan \frac{\sin \frac{(1-\mu) \pi}{2}+K_{d} \omega^{\mu}}{\cos \frac{(1-\mu) \pi}{2}}-\frac{(1-\mu) \pi}{2} \\
|C(j \omega)|=K_{p} \sqrt{\left(1+K_{d} \omega^{\mu} \cos \frac{\mu \pi}{2}\right)^{2}+\left(K_{d} \omega^{\mu} \sin \frac{\mu \pi}{2}\right)^{2}}
\end{gathered}
$$

Thus the open loop transfer function $L(s)$ can be shown as

$$
L(s)=C(s) G(s)
$$

It follows from (10) and (13) that

$$
\arg |L(j \omega)|=\arctan \frac{\sin \frac{(1-\mu) \pi}{2}+K_{d} \omega^{\mu}}{\cos \frac{(1-\mu) \pi}{2}}-\frac{(1-\mu) \pi}{2}-\arctan \left(\omega T_{m}\right)-\frac{\pi}{2}
$$

In frequency domain, there are three roles to design the fractional order $\mathrm{PD}^{\mu}$ control:

(1) Phase margin constraint

$$
\arg \left|L\left(j \omega_{c g}\right)\right|=\operatorname{agr}\left|C\left(j \omega_{c g}\right) G\left(j \omega_{c g}\right)\right|=-\pi+\phi_{m}
$$

where $\varphi_{\mathrm{m}}$ is the phase margin and $\omega_{\mathrm{cg}}$ denotes the cut-off frequency.

(2) Robustness constraint

$$
\left.\frac{d(\arg [L(j \omega)])}{d \omega}\right|_{\omega=\omega_{c g}}=0
$$

It should be noted that the zero derivative of $\mathrm{L}(\mathrm{j} \omega)$ to $\omega$ denotes the robustness of the closed control system so that the overshoots can be efficiently restricted for varying system gains. This is the core to design the fractional order robust control of Leader I-DX robot.

(3) Amplitude margin constraint

$$
\left|L\left(j \omega_{c g}\right)\right|=\left|C\left(j \omega_{c g}\right)\right|\left|G\left(j \omega_{c g}\right)\right|_{d b}=1
$$




\subsection{The Parameters of Fractional Order PD $^{\mu}$ Controller}

(1) Considering equation (17) we have

$$
\arg |L(j \omega)|=\arctan \frac{\sin \frac{(1-\mu) \pi}{2}+K_{d} \omega^{\mu}}{\cos \frac{(1-\mu) \pi}{2}}-\frac{(1-\mu) \pi}{2}-\arctan \left(\omega T_{m}\right)-\frac{\pi}{2}=-\pi+\varphi_{m}
$$

It follows from (20) that $K_{d}$ and $\mu$ are related as

$$
K_{d}=\frac{1}{\omega_{c g}{ }^{\mu}} \tan \left[\varphi_{m}+\arctan \omega_{c g} T_{m}-\frac{\mu \pi}{2}\right] \cos \frac{(1-\mu) \pi}{2}-\frac{1}{\omega_{c g}{ }^{\mu}} \sin \frac{(1-\mu) \pi}{2}
$$

(2) Using equation (18) we have

$$
\left.\frac{d(\arg (L(j \omega)))}{d \omega}\right|_{\omega=\omega_{c g}}=\frac{\mu K_{d} \omega_{c g}{ }^{\mu-1} \cos \frac{(1-\mu) \pi}{2}}{\cos ^{2} \frac{(1-\mu) \pi}{2}+\left(\sin \frac{(1-\mu) \pi}{2}+K_{d} \omega_{c g}{ }^{\mu}\right)^{2}}-\frac{T_{m}}{1+\left(T_{m} \omega_{c g}\right)^{2}}=0
$$

It follows from (22) that $K_{d}$ can be computed by

So that

$$
A \omega_{c g}^{2 \mu} K_{d}^{2}+B K_{d}+A=0
$$

$$
K_{d}=\frac{-B \pm \sqrt{B^{2}-4 A^{2} \omega_{c g}^{2 \mu}}}{2 A \omega_{c g}^{2 \mu}}
$$

where $A=\frac{T_{m}}{1+\left(T_{m} \omega_{c g}\right)^{2}}, B=2 A \omega_{c g}{ }^{\mu} \sin \frac{(1-\mu) \pi}{2}-\mu \omega_{c g}{ }^{\mu-1} \cos \frac{(1-\mu) \pi}{2}$.

(3) The constraint in equation (19) gives

$$
\left|L\left(j \omega_{c g}\right)\right|=|C(j \omega)||G(j \omega)|=\frac{K \cdot K_{p} \sqrt{\left(1+K_{d} \omega_{c g}{ }^{\mu} \cos \frac{\mu \pi}{2}\right)^{2}+\left(K_{d} \omega_{c g}{ }^{\mu} \sin \frac{\mu \pi}{2}\right)^{2}}}{\omega_{c g} \sqrt{1+\left(\omega_{c g} T_{m}\right)^{2}}}=1
$$

By doing so the $K_{d}$ and $\mu$ can be computed by combing (21) and (24). It should be noted that it is always a very heavy work to compute such parameters. In this paper, a practical strategy is proposed by using MATLAB. The detailed procedure is:

1) Given the desired cut-off frequency $\omega_{c g}$.

2) Given the desired phase margin $\varphi_{m}$.

3) Plot the curve in equation (21), where $\mu$ is a independent variable and $K_{d}$ is a dependent variable.

4) Plot the curve in equation (24), where $\mu$ is a independent variable and $K_{d}$ is a dependent variable. 
5) As shown in Figure 2, the intersection of the above two curves gives the values of $\mu$ and $K_{d}$.

6) Using equation (25) gives the value of $K_{p}$

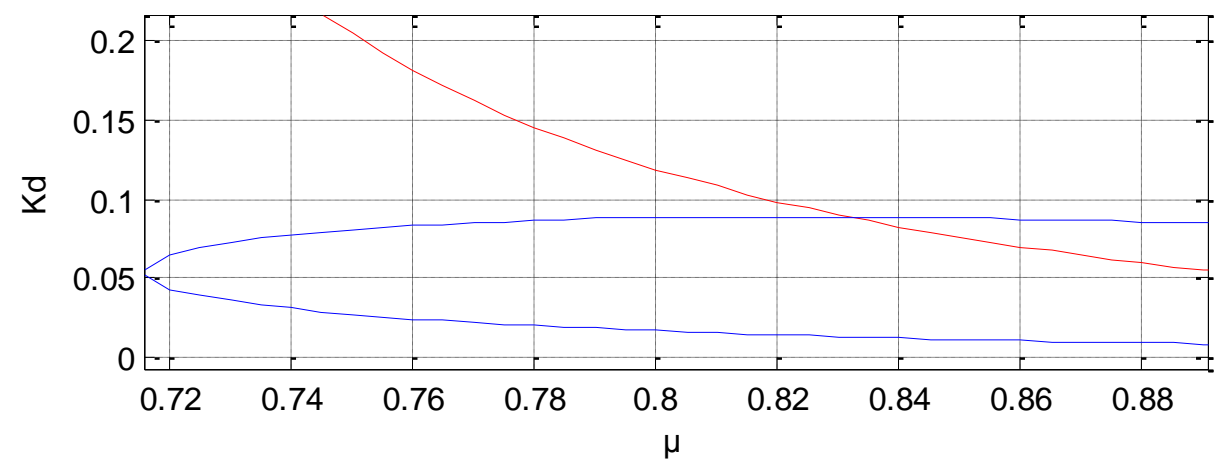

Figure 2. The Curves of $\mu$ and $K_{d}$

To our experiences, it is assumed that $\omega_{c g}=60 \mathrm{rad} / \mathrm{sec}$ and $\varphi_{m}=70^{\circ}$. It follows from the intersection in Figure 2 that $\mu=0.83$ and $K_{d}=0.09$. It then follows from (25) that $K_{p}=1.18$. Therefore, the fractional order $\mathrm{PD}^{\mu}$ control is

$$
c(s)=1.18\left(1+0.09 s^{0.83}\right)
$$

\section{The Control System and Simulation Results}

\subsection{The Analysis of Control Systems}

The system model of the DC motor of Leader I-DX is built in MATLAB/SIMULINK, which is shown in Figure 3.

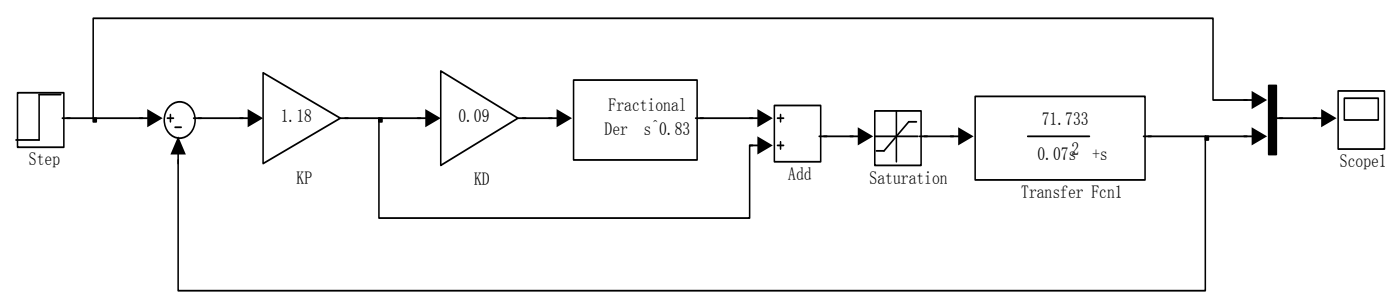

Figure 3. The System Model of Leader I-DX

The Bode plot of the open loop fractional order $\mathrm{PD}^{\mu}$ contrl system is shown in Figure 4. As shown in this figure, the designed fractional order $\mathrm{PD}^{\mu}$ controller not only satisfy the phase margin requirement but also remain unchanged in a certain range of the cut-off frequency $\omega_{c g}$. 


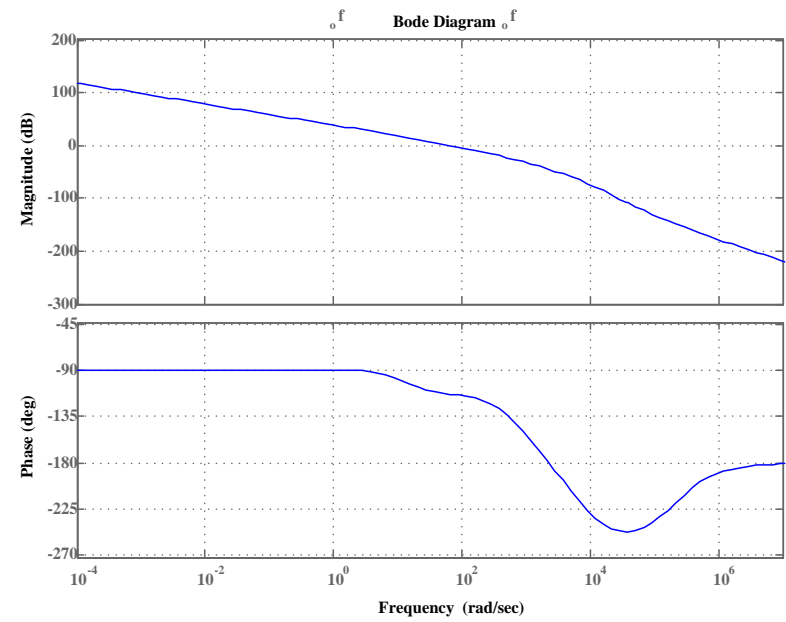

Figure 4. The Bode Plot of the Fractional Order PD $\mu$ Control System

\subsection{The Influence of $\mu$ to the Control System}

The step responses of the control system are shown in Figure 5. It can be seen that, for $\mu$ less than 0.83, the overshoot, oscillating, tuning time and static accuracy are all improved at the same time if $\mu$ is increased. A larger $\mu$ may deduce to slow tracking and low accurate static error. When $\mu$ is too small, there are too large overshoots, strong oscillating or even non-stability. Nevertheless, a small change of $\mu$ in a small field doesn't influence of performance of fractional order control system.

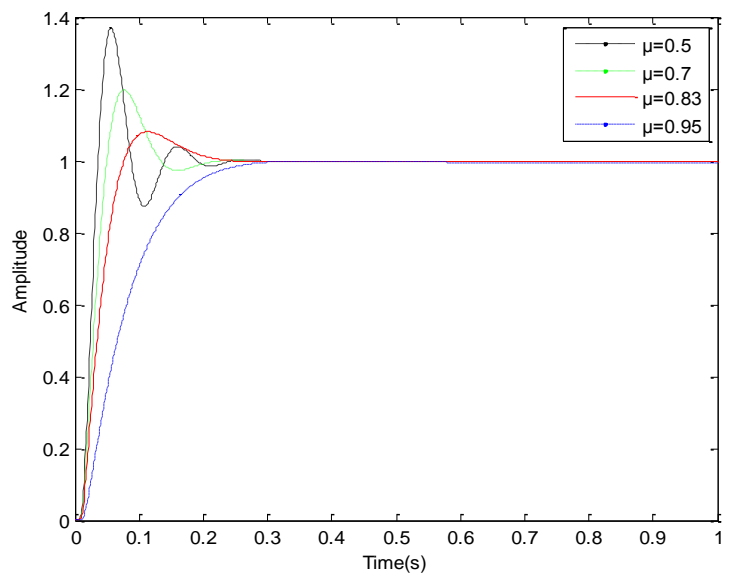

Figure 5. The Step Responses for Different $\mu$

\subsection{The Comparisons of $\mathrm{PD}^{\mu}$ and PD Controllers}

The comparisons of $\mathrm{PD}^{\mu}$ and $\mathrm{PD}$ controllers are shown in Figure 6. It can be seen that, for various $\mathrm{K}_{\mathrm{d}}$ in $\mathrm{PD}$ controller, the fractional order $\mathrm{PD}^{\mu}$ controller (dotted line) performs better than the integer order ones (bold lines), which implies the advantages of fractional order control. 


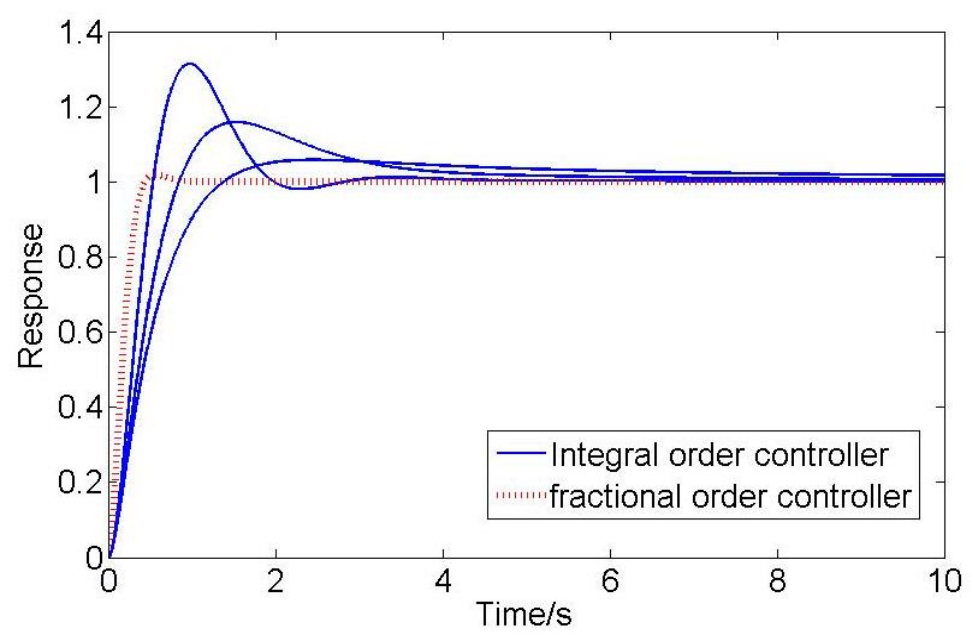

Figure 6. The Step Response Comparison between Fractional Order PD controller and Integral Order PD Controller

\section{The Digital Implementation of Fractional Order Control}

To further improve the robustness of fractional order control system, and consider the implementation of fractional order controllers, a low pass filter is cascaded to the fractional order derivative so that the fractional order controller is expressed as:

$$
C(s)=K_{P}\left(1+K_{d} \frac{s^{\mu}}{T s+1}\right)=K_{P}\left(1+K_{d} G_{0}(s)\right)
$$

where $\mathrm{T}=1, \mathrm{G}_{0}(\mathrm{~s})$ denotes the differentiator that implemented by adding a low pass filter. Besides, the inverse Laplace transform of $\frac{s^{\alpha-\beta}}{s^{\alpha}+\tau}$ is

$$
t^{\beta-1} E_{\alpha, \beta}\left(-\tau^{\alpha}\right)
$$

where $E_{\alpha, \beta}()$ is the Mittag-Leffler function in two parameters. It follows from (28) that the impulse response of $\mathrm{G}_{0}(\mathrm{~s})$ is

$$
h_{0}(t)=t^{1-\mu} E_{1,1-\mu}(-t)
$$

By using the PRONY function in MATLAB, the approximated discrete transfer function of $\mathrm{G}_{0}(\mathrm{~s})$ can be shown as

$$
\begin{gathered}
G_{0}(z)=\frac{-0.008747 z-0.001903}{z-1.019} \\
z U(z)-1.019 U(z)=K_{p}\left(z-1.019+K_{d}(-0.008747 z-0.001903)\right) E(z)
\end{gathered}
$$

Given the zero initial condition, the discrete fractional order $\mathrm{PD}^{\mu}$ control is

$$
\begin{aligned}
& u(k)=1.019 u(k-1)+K_{P}[e(k)-1.019 e(k-1) \\
& \left.+K_{d}(-0.008747 e(k)-0.0101903 e(k-1))\right]
\end{aligned}
$$


Thus the incremental fractional order $\mathrm{PI}^{\lambda} \mathrm{D}^{\mu}$ controller can be shown as

$$
\begin{aligned}
& \Delta u(k)=u(k)-u(k-1) \\
& =0.019 u(k-1)+K_{P}[e(k)-1.019 e(k-1) \\
& \left.+K_{d}(-0.008747 e(k)-0.0101903 e(k-1))\right]
\end{aligned}
$$

\section{Experiments}

Let the referential speed of Leader I-DX be $600 \mathrm{~mm} / \mathrm{s}$, and the sample instant be $8 \mathrm{~ms}$, the system output is shown in Figures 7 and 8. It can be seen that the fractional order PD ${ }^{\mu}$ control strategy has a faster tracking speed. Besides, too many oscillations are observed in the traditional PD control case. Moreover, it should be noted that the weight of Leader IDX is $50 \mathrm{Kg}$. Thus a slow response is happened for zero initial state, which can be improved by an initial excitation.

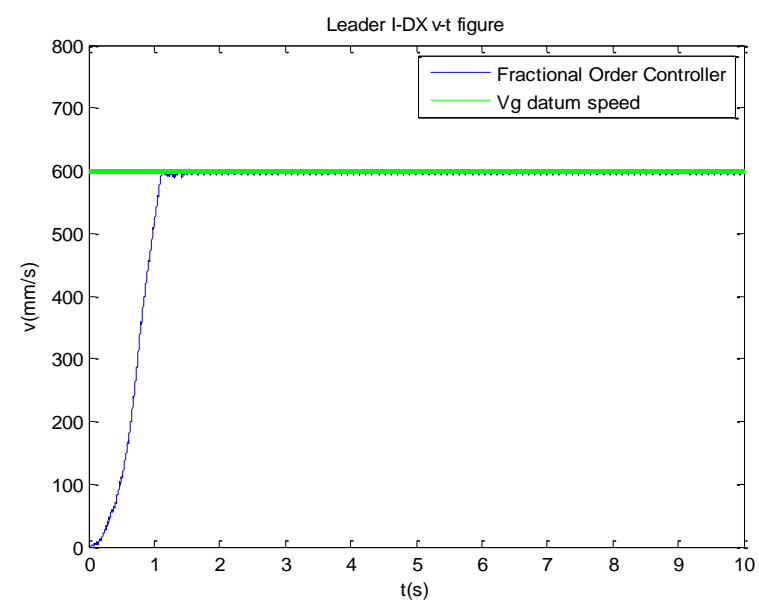

Figure 7. Fractional Order PD $^{\mu}$ Control

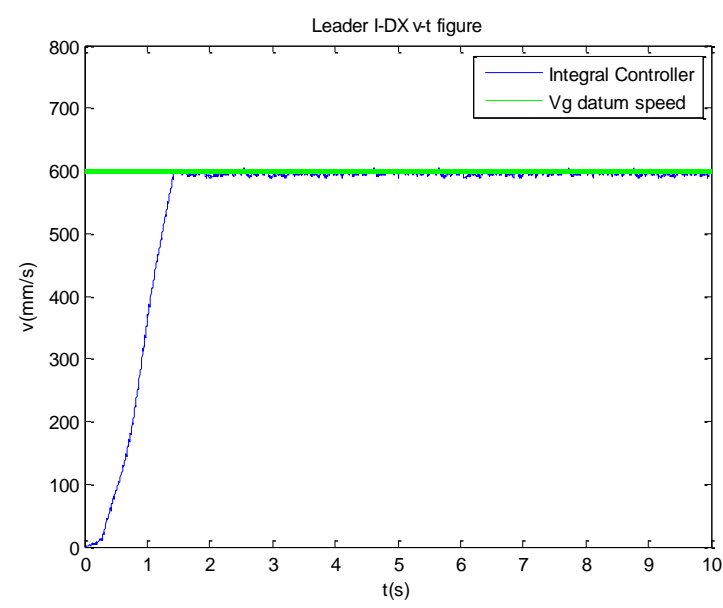

Figure 8. Integer Order PD Control

\section{Conclusions}

This paper discusses the robust design of fractional order $\mathrm{PD}^{\mu}$ control, which is applied to the speed tracking control of Leader I-DX service robot. It has been verified in simulations and experiments that the fractional order $\mathrm{PD}^{\mu}$ control performances better than 
traditional PD controllers, where the faster tracking speed and the smaller tracking error can be obtained.

\section{Acknowledgments}

This project is supported by the National Natural Science Foundation of China (No.61375084), the Key Subjects at the Provincial Level of Fujian Province[2012] No.136.

\section{References}

[1] I. Podlubny, "Fractional differential equations", San Diego: Academic Press, (1999).

[2] L. Debnath, "A brief historical introduction to fractional calculus", International Journal of Mathematical Education in Science and Technology, vol. 35, no. 4, (2004), pp. 487-501.

[3] P. J. Torvik and R. L. Bagley, "On the appearance of the fractional derivative in the behavior of real material", Journal of Applied Mechanics, Transaction of the ASMF, vol. 51, no. 2, (1984), pp. 294-298.

[4] B. Dumitru, Z. B. Güvenç, and J. A. Tenreiro Machado, "New Trends in Nanotechnology and Fractional Calculus Applications", Springer, (2010).

[5] J. Sabatier, O. P. Agrawal, and J. A. Tenreiro Machado, "Advances in Fractional Calculus: Theoretical Developments and Applications in Physics and Engineering", Springer, (2007).

[6] C. B. Ma and Y. Hori, "Fractional-Order Control: Theory and Applications in Motion Control", IEEE Industrial Electronics Magazine, vol. 1, no. 4, (2007), pp. 6-16.

[7] M. Axtelland and M. E. Bise, "Fractional calculus applications in control systems", In: Proceedings of the IEEE Nat. Aerospace and Electronics Conference. New York, USA, (1990), pp. 563-566.

[8] D. Xue and Y. Q. Chen, "Fractional order Calculus and Its Applications in Mechatronic System Controls Organizers", In: Proceedings of the 2006 IEEE International Conference on Mechatronics and Automation, (2006).

[9] C. A. Monje, B.M Vinagre and Y. Q. Chen, "Proposals for fractional $\mathrm{PI}^{\lambda} \mathrm{D}^{\mu}$ tuning. In: Proceedings of the first IFAC Symposium on Fractional Differentiation and its Applications (FDA04)", Bordeaux, France, (2004)

[10] B. M. Vinagre, C. A. Monje, A. J. Calderón, and J. I. Suárez, "Fractional PID controllers for industry application", A brief introduction. Journal of Vibration and Control, vol. 13, (2006), pp. 1419-1429.

[11] C. A. Monje, B. M. Vinagre, V. Feliuc, and Y. Q. Chen, "Tuning and autotuning of fractional order controllers for industry applications", Control Engineering Practice, vol. 16, (2008), pp. 798-812.

[12] A. Oustaloup, «La dérivation non entière: Théorie, synthèse et applications », Hermes Edition, Paris, France, (1995).

[13] L. Dorcak, "Numerical models for the simulation of the fractional-order control systems", arxiv preprint math/0204108, (2002).

[14] I. Podlubny, "Fractional-order systems and $\mathrm{PI}^{\lambda} \mathrm{D}^{\mu}$ controllers", IEEE Transactions on Automatic Control, vol. 44 , no. $1,(\mathbf{1 9 9 3})$, pp. 208-214.

[15] A. J. Calderón, B. M. Vinagre and V. Feliu, "Linear fractional order control of a DC-DC buck converter", In: Proceedings of European Control Conference, Cambridge, UK, (2003).

[16] I. Tejado, B. M. Vinagre and Y. Q. Chen, "Fractional Gain and Order Scheduling Controller for Networked Control Systems with Variable Delay", Application to a Smart Wheel. In: Proceedings of the 4th IFAC Workshop on Fractional Differentiation and Its Applications, University of Extremadura, Badajoz, Spain, (2010), October 18-20.

[17] Y. Q. Chen, "Fractional Calculus, Delay Dynamics and Networked Control Systems", In: Proceedings of the 3rd International Symposium on Resilient Control Systems, (2010), August 10-12, pp. 58-63.

[18] F. B. M. Duart and J. A. T. Machado, „Pseudoinverse trajectory control of redundant manipulators: a fractional calculus perspective", In: Proceedings of International Conference on PRobotics and Automation. Washington, 2002, 175-180.

[19] X. Wang, "Reseach and Design of Leader I-DX Autonomous Mobile Platform for Service Robot", Jinan: Shandong University, (2010). 


\section{Authors}
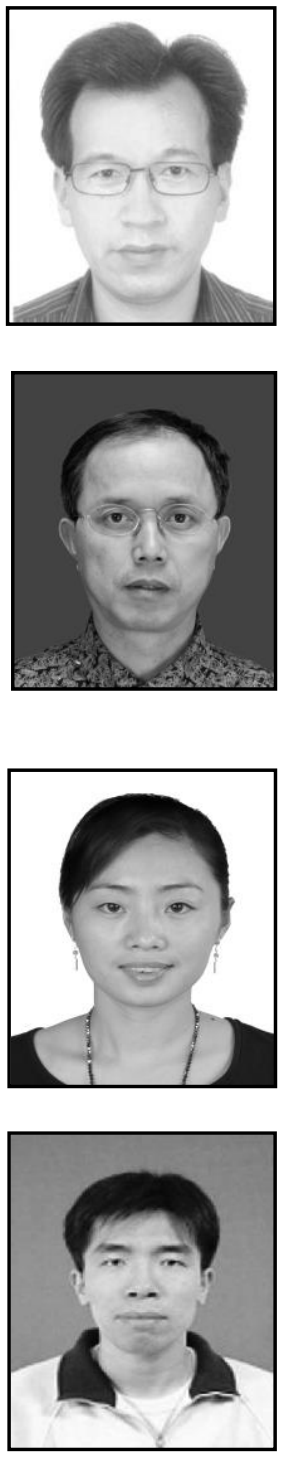

Li Guodong, he was born on October 17, 1981. He is a man. He is from Jinan, China. He is a lecturer in Longyan University. His research interests include machine vision, intelligent control and visual servo.

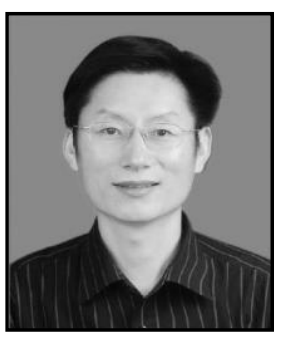

Zhou Fengyu, he was born on march 8,1969 . He is a man. He is from Linyi, China. He is a professor and $\mathrm{PhD}$ supervisor in Shandong University. His research interests include robotics, intelligent instrument, intelligent control theory and computer control system. 
International Journal of Control and Automation Vol.8, No.5 (2015) 